\title{
Bacterial cooperation controlled by mobile elements: kin selection versus infectivity
}

Heredity (2011) 107, 277-278; doi:10.1038/hdy.2011.57; published online 27 July 2011

In a recent paper, Rankin et al. (2010) review which traits are carried on mobile genetic elements (MGEs) and why. Much of the review is dedicated to a recent paper (Nogueira et al., 2009) exploring the mechanisms that can maintain cooperative traits coded by MGEs in pathogenic bacteria, for example, the secretion of toxins into the environment, which are costly to individuals that bear them but benefit the local population at large, because they allow host infection. They argue that horizontal transfer of MGEs in bacteria promotes cooperation by a kin selection mechanism because transmission increases genetic relatedness at MGEs encoding costly toxins. Here we outline three reasons why their evidence is not sufficient to support kin selection maintaining altruism in bacteria carrying cooperative MGEs over the mere property of infectivity of these plasmids (Smith, 2001).

\section{The problem of kin selection in a structured population}

Understanding the maintenance of cooperation is of fundamental importance in evolutionary biology. Kin selection is a powerful mechanism that explains altruism (Hamilton, 1964), the principle being that preferential cooperation among relatives prevents cheaters from benefiting from altruism without paying its cost. Preferential cooperation among relatives indeed ensures that the benefits of cooperation go to individuals having a high probability of also carrying the altruistic allele. The production of costly common goods in particular is vulnerable to exploitation by cheaters. Understanding their maintenance is especially important when the common goods are toxins allowing virulence of pathogenic bacteria, because of implications for human health. Kin selection can drive the spread of altruistic traits through preferential cooperation among relatives (Hamilton, 1964; Grafen, 1985; Gardner and West, 2010), that is, if cooperation occurs among individuals with a higher genetic similarity than average in the population at the cooperation locus, which is usually translated as having a relatedness $R>0$. In their review, Rankin et al. (2010) argue that horizontal transmission of MGE carrying altruistic traits, for example, the secretion of toxins into the environment, increase relatedness at this locus within populations, and thus that kin selection can operate to maintain cooperation.

Altruism can thus be directed preferentially towards relatives in toxin-producing bacteria that occur in viscous populations. In structured populations, genetic similarity may indeed be high within patches compared with the population as a whole, such that cooperative bacteria interact more often with other cooperative bacteria than if the populations were mixed. Population viscosity has been proposed to promote altruism for this reason, but only under the condition that cooperation occurs within patches while competition for resources occurs between patches (Queller, 1994; Griffin and West, 2002; Pedersen et al., 2006). Indeed, if kin cooperate, but also compete for resources, the advantages of cooperation are cancelled.

Rankin et al. (2010) and Nogueira et al. (2009) consider a population structured in distinct patches, and show that relatedness increases within patches due to MGE transmission, but they fail to discuss where competition occurs. For their arguments to work, competition for resource acquisition in bacteria must occur between and not within patches. If the 'economic neighbourhood' were the patches for bacteria producing toxins as common goods, relatedness must compare genetic similarity between altruists and bacteria benefiting from toxins with genetic similarity between altruists and random bacteria chosen within a patch. Then, unless altruistic bacteria preferentially cooperate with other altruistic bacteria within patches (as would be the case for instance if the plasmid encoded a toxin to which plasmid carriers would be immune), relatedness between altruists and bacteria benefiting from toxins would be zero. For pathogenic bacteria, it is most likely that at least some competition occurs within patches as parasites compete over a common pool of host resources. Therefore, even high relatedness within patches may not allow kin selection to act on trait evolution because of the effects of local resource competition.

\section{What comes first: Cooperation or plasmid transmission?}

Another issue is that Rankin et al. (2010)'s model does not explicitly denote when cooperation occurs relative to plasmid transfer. If, for instance, toxins produced before host infection allow host colonization, followed by MGE transfer within hosts to plasmid-free bacteria, the toxinproducing bacteria would have helped bacteria not carrying the plasmid, and therefore, MGEs would not have preferentially helped copies of themselves for host infection. Even if the MGEs are subsequently transmitted to bacteria within the host, increasing relatedness at the end, this increase in MGE frequency would be due to its infectious properties, not from kin selection. Therefore here, again, obtaining high relatedness within patches for an infectious cooperative plasmid provides insufficient evidence that its frequency increased via kin selection over simple infection dynamics. However, kin selection may act if plasmids are first transferred among bacteria within hosts, and only then toxins are produced that allow more efficient use of host resources or avoidance of the host immune system (provided that competition 
among kin does not cancel the benefit of cooperation as outlined above).

\section{Evidence from genomic data}

In the original paper, Nogueira et al. (2009) used two lines of evidence in addition to their model to support their contention that kin selection maintains costly common goods in bacterial populations: 1) genes coding for secreted proteins, that is, candidates for cooperative traits, are more likely to be mobile than are other classes of genes; 2) secreted proteins are cheaper to produce than other proteins, thereby reducing $\mathrm{c}$ and rendering less stringent the conditions for evolution via kin selection whereby $\mathrm{Rb}>\mathrm{c}$. These results are highly interesting and certainly deserve explanation. However, neither supports kin selection over simple infection dynamics of mobile elements as the mechanism maintaining costly common goods. For the first, those genes for costly common goods that occur on mobile elements will be more likely to persist in bacterial populations simply because they can infect new lineages. For the second, common goods production will be more easily maintained if its cost is lower, regardless of the mechanism involved. Neither result supports kin selection over simple infection dynamics.

\section{Conclusion}

We conclude that Rankin et al. (2010) and Nogueira et al. (2009) provide no definitive evidence for kin selection acting in addition to MGEs' infectivity as a mechanism maintaining altruism in cooperative bacteria. We do not deny that altruism among pathogens could evolve by kin selection and there are examples (Koskella et al., 2006; Buckling et al., 2007; Lopez-Villavicencio et al., 2007 , 2011). It may well even be that infectivity evolves via kin selection in toxin-producing bacteria, but we contend that the lines of evidence brought in the paper of Rankin et al. (2010) and Nogueira et al. (2009) are far from sufficient to be conclusive. It is critical to precisely define the conditions under which kin selection could indeed help maintain altruism in these cases, and whether these conditions are met in real cases. In particular, it is essential to assess when and where competition takes place compared with cooperation and plasmid transmission.

\section{Conflict of interest}

The authors declare no conflict of interest.

T Giraud ${ }^{1,2,3}$ and JA Shykoff ${ }^{1,2,3}$

${ }^{1}$ University of Paris-Sud, Laboratoire Ecologie, Systématique et Evolution, UMR8079, Orsay, France; ${ }^{2}$ CNRS, Orsay, France and ${ }^{3}$ AgroParisTech, Orsay, France.

E-mail: Tatiana.Giraud@ese.u-psud.fr

\section{References}

Buckling A, Harrison F, Vos M, Brockhurst MA, Gardner A, West SA et al. (2007). Siderophore-mediated cooperation and virulence in Pseudomonas aeruginosa. FEMS Microbiol Ecol 62: 135-141.

Gardner A, West SA (2010). Greenbeards. Evolution 64: 25-38.

Grafen A (1985). A geometric view of relatedness. Oxford Surv Evol Biol 2: 28-89.

Griffin AS, West SA (2002). Kin selection: fact and fiction. Trends Ecol Evol 17: 15-21.

Hamilton WD (1964). The genetical evolution of social behavior. I. J Theor Biol 7: 1-16.

Koskella B, Giraud T, Hood ME (2006). Pathogen relatedness affects the prevalence of within-host competition. Am Nat 168: 121-126.

Lopez-Villavicencio M, Courjol F, Gibson A, Hood M, Jonot O, Shykoff JA et al. (2011). Competition, cooperation among kin and virulence in multiple infections. Evolution 65: 1357-1366.

Lopez-Villavicencio M, Jonot O, Coantic A, Hood M, Enjalbert J, Giraud T (2007). Multiple infections by the anther smut pathogen are frequent and involve related strains. PloS Path 3: e176.

Nogueira T, Rankin DJ, Touchon M, Taddei F, Brown SP, Rocha EPC (2009). Horizontal gene transfer of the secretome drives the evolution of bacterial cooperation and virulence. Curr Biol 19: 1683-1691.

Pedersen J, Krieger M, Vogel V, Giraud T, Keller L (2006). Supercolonies of unrelated individuals in native populations of the invasive Argentine ant. Evolution 60: 782-791.

Queller DC (1994). Genetic relatedness in viscous populations. Evol Ecol 8: 70-73.

Rankin DJ, Rocha EPC, Brown SP (2010). What traits are carried on mobile genetic elements, and why? Heredity 106: $1-10$.

Smith J (2001). The social evolution of bacterial pathogenesis. Proc Royal Soc London Series B-Biol Sci 268: 61-69. 